\title{
LE GHETTO DANS LE ROMAN ROUMAIN DE L'ENTRE-DEUX-GUERRES
}

Le roman urbain roumain, qui s'intéresse aux espaces marginaux tels que le bidonville, le quartier, la périphérie, les banlieues, n'est pourtant pas le seul à les explorer : le roman des mystères, plus tard celui des mœurs, puis le roman réaliste disposeront du même topos. La métropole, dont le processus rapide de modernisation a été observé dans la littérature dès le début du $\mathrm{XX}^{\mathrm{e}}$ siècle, se révèle, non seulement pour les sociologues, mais pour les écrivains aussi, comme « organisme», pas comme "création humaine inerte " dans le roman des décennies suivantes, les années 1930-1950, par G.M. Zamfirescu (Maidanul cu dragoste [Le terrain vague avec amour], 1933) et Eugen Barbu (Groapa [La fosse], 1957); les communautés périphériques représentées dans les romans, des faubouriens aux allogènes, révèlent des typologies et des interrelations singulières qui pourraient bien être influencées par la culture et la moralité urbaines, mais aussi l'influencer, à leur tour.

Sélectionnée pour son impact métonymique, l'image de la rue devient un symbole de la ville par excellence: de Cezar Petrescu à George Călinescu, l'Avenue de la Victoire de Bucarest exprime, dans de nombreux romans de la première moitié du $\mathrm{XX}^{\mathrm{e}}$ siècle, l'essence même du centre : "le creuset de la noblesse de premier rang de Bucarest $»^{2}$. Lorsqu'un autre romancier, I. Peltz, entend redonner à une communauté " fermée » de la visibilité en prose, il oppose l'image d'une rue apparemment dénuée de symbolisme, l'Avenue Văcărești, au même "espace privilégié par excellence », de l'Avenue de la Victoire. "Pour le lecteur peu initié, et même pour le bon lecteur, avoue l'écrivain dans une interview, l'Avenue Văcărești représente un quartier ordinaire, qui s'ouvre à la plaisanterie ou à l'ironie. L'Avenue de la Victoire est bien connue par l'Avenue Văcărești ; l'Avenue Văcărești, en revanche, est ignorée par l'Avenue de la Victoire et pas seulement par elle, mais par tout le monde $»^{3}$. I. Peltz s'engage à une radiographie

\footnotetext{
${ }^{1}$ Robert E. Park, « Prefață » [Préface], in Louis Wirth, Ghetoul [Le Ghetto]. Traduit par Lăcrămioara Juverdeanu. Étude introductive de Mihai Dinu Gheorghiu, Iași, Editura Universității « Al. I. Cuza », 2012, p. 35.

${ }^{2}$ Ovidiu Morar, Scriitori evrei din România [Ecrivains Juifs de Roumanie], București, Hasefer, 2014, p. 122.

${ }^{3}$ Camil Baltazar, «De vorbă cu d-1 I. Peltz, cu prilejul apariției romanului Calea Văcărești» [« Entretien avec M. I. Peltz, à l'occasion de la publication du roman L'Avenue Văcărești »], România literară $, 2,1933,87$, p. 3 .
} 
complète de l'Avenue Văcărești, ouvrant, en 1933, la série des romans qui mettra en premier plan une communauté minoritaire, qui venait d'être incluse légalement dans la nation roumaine depuis peu de temps ${ }^{4}$, la communauté juive. Le romancier fixe ainsi l'image de l'artère emblématique du quartier juif de Bucarest, en utilisant, à l'intérieur de l'intrigue, le réseau complexe de rues des quartiers Văcărești-Dudești ${ }^{5}$, dans une reconstitution monographique de la communauté ellemême. Et même si on peut délimiter sans effort les quartiers sensibles à l'antisémitisme chez Peltz, sa cartographie conserve, tout de même, des frontières lâches, minées par la perméabilité ethnique ${ }^{6}$ et par la mobilité de ses membres ${ }^{7}$. Ainsi, dans le ghetto illustré par l'écrivain roumain, deux des éléments considérés comme représentatifs dans la conception de Loïc Wacquant sont annulés : l'augmentation de l'homogénéité ethnique et les frontières imperméables 8 .

A comparer les définitions offertes par deux dictionnaires ${ }^{9}$ de l'entre-deuxguerres, on constate qu'on ne considère pas le ghetto comme une réalité roumaine, mais comme une réalité étrangère, italienne ou orientale (Lazăr Șăineanu), alors que la définition proposée par August Scriban insiste, une décennie plus tard, sur le caractère volontaire de l'agglomération qui caractérise le quartier juif.

Cela explique probablement pourquoi le concept de " ghetto » reste assez usité dans le texte littéraire : il est présent dans la prose roumaine de la troisième décennie du $\mathrm{XX}^{\mathrm{e}}$ siècle seulement dans deux titres (Ury Benador, Ghetto Veac XX [Ghetto XX Siècle] et I. Ludo, Ghettouri [Ghettos]). Ici, de même, le ghetto renvoie à sa signification historique ${ }^{10}$, générale ${ }^{11}$, difficile à repérer sur le plan local, plutôt métaphoriquement associée à une réclusion sentimentale, chez I. Peltz et Ury Benador. Jusqu'aux valences atroces qu'il va prendre la décennie suivante,

\footnotetext{
${ }^{4}$ L'émancipation de la population juive se produit à la fin de la Première Guerre mondiale, par le Traité des minorités (1919), suivie de son inscription dans la Constitution roumaine, en 1923.

${ }^{5}$ Les rues Lazăr, Olteni, Traian, Carol, auxquelles correspondront, en miroir, Lipscani, Smârdani, Gabroveni, Bărăție, Sf. Apostoli, du roman Foc în Hanul cu Tei [Feu à l'Auberge aux Tilleuls], de 1934. L'information devient encore plus précieuse dans le contexte où la zone est fortement affectée par les démolitions de la période communiste.

${ }^{6}$ La communauté des quartiers évoqués est plutôt multiculturelle, l'auteur rajoutant à la majorité juive des personnages d'origine roumaine, grecque, tsigane, albanaise.

${ }^{7}$ Camelia Crăciun, «Représentations de la vie et de l'espace juifs dans la littérature roumaine de l'entre-deux-guerres. Le Bucarest juif reflété dans les œuvres d'Isac Peltz », Etudes Balkaniques, 2010, 17, pp. 93-94

8 Loïc Wacquant, «Repenser le ghetto. Du sens commun au concept sociologique », Idées économiques et sociales, 2012, 167, p. 18.

9 Lazăr Șăineanu, Dicționar universal al limbei române [Dictionnaire universel de la langue roumaine], IV ème édition, București, Scrisul Românesc, 1929 ; August Scriban, Dicționarul limbii românești [Le dictionnaire de la langue roumaine], București, Institutul de Arte Grafice "Presa Bună », 1939.

${ }^{10}$ I. Ludo, Ghettouri [Ghettos], București, « Cartea », 1939, p. 21.

${ }^{11}$ Ibidem, p. 9.
} 
le ghetto de I. Peltz se rapproche sensiblement de ce que Loïc Wacquant appelle le "quartier ethnique», dont les habitants étaient liés plus par des "affinités culturelles et une concentration socioprofessionnelle » que par « des préjugés et des discriminations inflexibles $\gg{ }^{12}$. Des études démographiques identifient, à partir de 1878 et jusqu'à la fin du XIX ${ }^{\mathrm{e}}$ siècle, à Bucarest, pas moins que cinq de ces zones avec une concentration considérable de population juive ${ }^{13}$, admettant, cependant, l'existence de cette population en dehors des zones d'intérêt résidentiel et commercial. I. Peltz, à son tour, fait mention des rues vers lesquelles se dirige la bourgeoisie juive ${ }^{14}$.

Assumée par l'engagement de l'auteur de rester authentique, l'option de Peltz pour Văcărești-Dudești, l'un des quartiers résidentiels les plus pauvres, est naturelle, le quartier représentant son lieu de naissance. Si l'identification du quartier juif avec les banlieues roumaines, et, dans une certaine mesure, avec les « banlieues de partout $\rangle^{15}$, est, selon certains chercheurs, la preuve claire de son acculturation $^{16}$, pour Peltz, le terme reste inapproprié, ne couvrant pas la complexité existentielle de sa communauté (« les profondeurs de la vie »).

Affirmant à un coreligionnaire à propos de l'Avenue Văcăreşti que ce ne serait pas «seulement du faubourg juif, mais de la vie ici, dans le ghetto », Peltz ne se contredit qu'en apparence ${ }^{17}$. Son ghetto n'est pas de jure, les réalités vont malheureusement changer seulement dans quelques années ${ }^{18}$, mais de facto, le terme de «faubourg» n'ayant pas la capacité d'exprimer sémantiquement les dommages moraux et les conséquences psychiques d'une telle coexistence, bien que la pauvreté et la marginalisation restent des dérivés constants dans le cas de la banlieue comme du ghetto. Le panoptique des personnages de I. Peltz du roman L'Avenue Văcărești reste, à cet égard, impressionnant :

L'Avenue Văcărești rassemble ses habitants. Du lointain du centre, ils retournent dans les rues étroites, débordantes de ténèbres et de mystère, de hauts boutiquiers couronnés de souffrance, des filles minces en blouses légères, étonnées et fatiguées, des vieux jetons, torturés au tour de taille, des matrones aussi vastes que des armoires... Les salons de thé se sont rallumés une fois de plus... Les garçons aux longs

\footnotetext{
${ }^{12}$ Loïc Wacquant, « Repenser le ghetto... », p. 23.

${ }^{13}$ Radu Ștefan Vergatti, «Dinamica demografică și aspecte socio-profesionale ale obștii evreiești din București (1810-1939) » [« La dynamique démographique et quelques aspects socioprofessionnels de la communauté juive de Bucarest (1810-1939) »], Revista de Istorie Socială, 1997-1998, 2-3, pp. 184-185. 14 I. Peltz, L’Avenue Văcărești, București, ESPLA, 1957, p. 276: «Ces Juifs n’habitaient pas vraiment l'Avenue Văcărești ou les rues du quartier. Ils s'étaient échappés vers le centre, vers Doamnei, Smârdan, Șelari, Rahovei, et même vers Batiște et Polonă ».

${ }^{15}$ Camil Baltazar, « De vorbă... », p. 3.

${ }^{16}$ Camelia Crăciun, « Représentations de la vie... », p. 94.

${ }^{17}$ Camil Baltazar, « De vorbă... », p.3.

${ }^{18}$ Sur se sujet, voir le numéro thématique de la Revue d'Histoire de la Shoah, 194, 2011, 1, «L'horreur oubliée : La Shoan roumaine ».
} 
cheveux flottant sur les épaules sont revenus, les yeux minés par les rêves, aux lèvres livides, perdus dans leur totale rêverie, pour assouvir leur faim d'autre chose à la table tachée pendant des années... Ils vont façonner, comme hier, comme avant-hier, comme toujours, le nouveau monde... Devant les tavernes aux grilles et aux violons, des mendiants se figeaient dévastés par les années avalées avec difficulté; ils gémissent sur leurs orbites en se grattant les mains. Bouches hilaires, fronts tordus, jambes tremblantes - L'Avenue Văcărești est un immense panoptique imaginé par un esprit échappé des brides. Il y a des âmes ici, vers lesquelles, pour les approcher, il faut monter quelques marches ; il y en a d'autres auxquelles, pour les comprendre, il faut descendre des marches... L'amertume grandit en lui, il le noie. La vie, dans L'Avenue Văcărești, est affectée par les mites ${ }^{19}$.

Cependant, ce qui était considéré comme le résultat d'une «discrimination typologique $»^{20}$, la galerie des « saints et martyrs » de Peltz, contrairement à celle des stéréotypes sémitiques, marque le début de la longue confession sans ressentiment des écrivains juifs «sur les juifs ». Peltz étant le premier à assumer l'impartialité, désirant être « un stylo qui écrit à froid $»^{21}$. Chez Ion Călugăru, G. Călinescu appréciera la même objectivité sans « douceur » ${ }^{22}$ dans la restitution du monde juif dans le roman Viața unui netrebnic [L'enfance d'un vaurien], tandis que Ury Benador donne, avec Ghetto Veac XX [Ghetto XX Siècle], «le livre hébreu le plus typique» qui est apparu «dans la littérature roumaine, depuis Manasse de Ronetti Roman $»^{23}$.

Entre le moment du doute sur l'existence d'une littérature juive de langue roumaine ${ }^{24}$ et l'apparition du volume qui ouvre la suite des romans représentatifs sur le ghetto (I. Peltz, Calea Văcărești, 1933), deux années se succèdent. Jusqu'à la montée politique de la droite radicalisée, aboutissant à l'ostracisme de la population juive, et culturellement, à l'indexation de sa création littéraire, apparaîtra Foc în Hanul cu Tei [Feu à l'Auberge aux Tilleuls] (1934) du même I. Peltz, Ghetto XX Siècle de Ury Benador et L'enfance d'un vaurien d'Ion Călugăru (1936). Depuis L'Avenue Văcărești d'une métropole en pleine émancipation de la Grande Roumanie (Bucarest), en passant par le quartier juif d'une importante ville du Danube (Brăila), à la «ruelle de Tipra» d'un anonyme bourg moldave (Dorohoi), le roman roumain du ghetto tend à compléter la fresque de la communauté juive de l'entre-deux-guerres à contretemps, phénomène dont l'envergure reste à deviner à partir des projets littéraires entamés, certains

\footnotetext{
${ }^{19}$ I. Peltz, Calea Văcărești, p. 329.

${ }^{20}$ G. Călinescu, Istoria literaturii române de la origini până în prezent [L'Histoire de la littérature roumaine dès origines jusqu'au présent]. Édition et preface d' Al. Piru, București, Minerva, 1982, p. 793.

${ }^{21}$ Camil Baltazar, « De vorbă... », p. 3.

${ }^{22}$ G. Călinescu, Istoria..., p. 795.

${ }^{23}$ Pompiliu Constantinescu, Scrieri [Écrits], I, București, Editura pentru Literatură, 1967, p. 203.

${ }^{24}$ A.L. Zissu, «Literatura evreiască din România » [« La littérature juive en Roumanie »], Adam, 3, 1931, 38, pp. 10-12.
} 
abandonnés après le premier volume. De la trilogie de Ury Benador, Ghetto Veac $X X$, seul le volume Germinări [Germinations] ${ }^{25}$ apparaît, sans Mâl şi beznă [Vase et ténèbres] et Urcuş în gol [Montée vide]. D'autres projets littérares ont été finalisés : dans le prolongement de l'Enfance d'un vaurien, Ion Călugăru signe également Trustul [Le Trust $]^{26}$ et Lumina primăverii [La lumière du printemps $]^{27}$.

L'inédit de la démarche des écrivains juifs de langue roumaine ne le représente pas autant la source d'inspiration, la communauté « contribuant», bien avant, à la mise en place dans la littérature populaire de vigoureux stéréotypes ${ }^{28}$, que l'hypostase de raconter cette communauté de l'intérieur, du milieu presque inconnu et contraignant pour la plupart des lecteurs. L'entretien de Peltz avec Camil Baltazar, juste avant la publication de son roman, en 1933, laisse voir comment il conçoit son lecteur ${ }^{29}$, et rend explicite, en même temps, son objectif : lutter contre les stéréotypes ${ }^{30}$, point qui se retrouve dans la poétique des autres écrivains juifs en Europe $^{31}$. Des engagements tels que le rejet de l'artificiel, l'objectivité, l'expérience personnelle guident la démarche narrative de l'écrivain juif dans le découpage temporel de la troisième décennie : vu dans son ensemble le roman du ghetto convainc d'abord par son caractère biographique. Buiumaș (L'Enfance d'un vaurien), Baruch Landau (Ghetto XX Siècle), Ficu (L'Avenue Văcărești) sont des protagonistes et, en même temps, des alter ego des écrivains, de multiples similitudes avec leurs biographies étant repérées par des histoires littéraires et des monographies: des noms et des années de naissance, chez Ion Călugăru, des familles entières, chez Isac Peltz, tout descend directement de la réalité du ghetto dans la fiction. D'ailleurs, Peltz avait affirmé dans une interview de la revue Rampa qu'il avait rencontré, personnellement, «tous les héros» de son roman ${ }^{32}$, après avoir même présenté, auparavant, à Cicerone Theodorescu, un d'entre eux ${ }^{33}$.

\footnotetext{
${ }^{25}$ Le roman connaît trois éditions : 1934, 1937 et 1947.

${ }^{26}$ Ion Călugăru, Trustul [Le Trust], [București], Editura « Națională Ciornei », [1937].

${ }^{27}$ Ion Călugăru, Lumina primăverii [La lumière du printemps], București, Socec, [1939].

${ }^{28}$ Andrei Oișteanu, Imaginea evreului în cultura română. Studiu de imagologie în context est-centraleuropean [L'image du Juif dans la culture roumaine. Étude d'imagologie dans un contexte d'Europe centrale et orientale], III ${ }^{\mathrm{ème}}$ édition, révisée et illustrée, Iași, Polirom, 2012.

${ }^{29}$ Camil Baltazar, «De vorbă... », p. 3 : «à mes amis connus et inconnus, de Bucarest et Vințul de Jos, de Ploiești et Sighetul Marmației, d'Odorhei et de Storojineț ».

30 Ibidem: "Je voulais montrer à tout le monde [...] que le Juif n'est pas seulement un objet d'anecdote, que son type n'est pas monopolisé par le courtier bavard du café ou le marchand obèse de tous les centres commerciaux $»$.

31 Nadia Malinovich, «Littérature populaire et romans juifs dans la France des années 1920 », Archives Juifs, 39, 2006, 1, p. 50.

32 I. Podeanu, « Douăzeci de minute cu d. I. Peltz » [« Vingt minutes avec M. I. Peltz »], Rampa, 17, 1934, 5001, p. 1.

${ }^{33}$ Iacob Saltzman, alias Șulăm Șolț, in Cicerone Theodorescu, Cu I. Peltz pe Calea Văcărești..., p. 721.
} 
Pour beaucoup de personnages de la communauté juive, la perception du ghetto comme un «micro-univers [...] fermé et suffisant à lui-même $»^{34}$ limite souvent l'action du roman à un espace exclusif, de sorte que cette " unité de lieu » s'impose comme une particularité ; d'autre part, le ghetto synthétise, en substance, le même déracinement des personnages, et à cet égard il peut être considéré comme identique à n'importe quel autre ghetto évoqué par Peltz, Călugăru ou Benador. Le personnage de ce dernier, Mendl, évoque, à la veille de son départ pour une nouvelle destination, qui est toujours un ghetto, le pèlerinage infatigable de la nation :

Il aimerait dormir aussi, mais son sommeil ne colle pas à ses yeux : ainsi, la route mène en descente, de plus en plus en bas. Mon père, de Sadagura à Tchernivtsi et Rădăuți, puis de Rădăuți à Dorohoi et maintenant à Brăila » ${ }^{35}$, un voyage repris par son fils, Baruch Landau, à Bucarest, et, enfin, en Palestine, considérée comme «le début du chemin du retour vers le premier Canaan ${ }^{36}$.

Si pour les personnages adultes l'évasion se fait en substituant un ghetto par un autre, se sauver par la nature est la solution trouvée par les personnages des jeunes : Buiumaș l'utilise, mais aussi l'adolescent Baruch :

Sans un but, il part en avant, loin jusqu'au bout, puis repart à droite, sur la ligne de tramway sortant de la ville jusqu'à ce qu'il entre dans la jeune forêt du Monument. Ici, sur le banc où il est assis, il s'est assis peut-être tant d'années avant Ștefan Petică $\breve{~}^{37}$, le poète des vierges en blanc. Par sa simple évocation, il se ressuscite. Et lui aussi, Baruch Landau, revivra dans la mémoire de je ne sais quels rêveurs, au fil des décennies et des siècles. Ici, dans cet espace, se trouvait la tête de Ștefan Petică. Regarde ici. Il s'était allongé sur le banc face au soleil. Pourquoi ses yeux lui font-ils mal ? Il les ferme. Il restera comme ça toujours, toujours. Sans pensées, sans soucis ${ }^{38}$.

Présence rare dans la métropole de Bucarest, la nature résonne trop peu avec l'idée du refuge salutaire: on refuse à la malade Esther même la joie de la contempler à travers la fenêtre, et à la beauté gratuite du petit jardin fleuri de Țipra (la mère de Buiumaș), on ne peut opposer que le minuscule, mais rentable vignoble, le coing et le prunier de Șulăm Șolț. Dans le périmètre surpeuplé du ghetto, l'espace vert, inévitablement associé au bien-être, est, pour cette raison, quasi inexistant. Pourtant, la nature hante les souvenirs des personnages de

\footnotetext{
${ }^{34}$ M. Bahtin, Probleme de literatură și estetică [Problèmes d'esthétique et de littérature]. Traduit par Nicolae Iliescu. Préface de Marian Vasile, București, Univers, 1982, p. 455.

${ }^{35}$ Ury Benador, Ghetto Veac XX [Ghetto XX $X^{e}$ Siècle], București, Editura Librăriei «Universala » Alcalay\&Co, [1934], p. 39

${ }^{36}$ Ibidem, p. 5.

${ }^{37}$ Ștefan Petică (1877-1934), poète symboliste, journaliste, dramaturge. Le volume Fecioara în alb [La vièrge en blanc], publié en 1902, contient les cycles : Fecioara în alb [La vièrge en blanc], Când vioarele tăcură [Quand les violons se sont tus], Moartea visurilor [La mort des rêves].

${ }^{38}$ Ury Benador, Ghetto Veac XX, pp. 96-97.
} 
L'Avenue Văcărești, dans le regret d'Esther de ne pas avoir installé sa maison «quelque part à la campagne» ou dans «le parfum d'une époque éteinte : des parcs urbains, avec des filles contournant les ruelles d'automne».

Faisant partie de la réalité urbaine, le ghetto surprend la ville dans des hypostases et des étapes distinctes: métropole multiethnique en pleine émancipation (Bucarest), prospère ville portuaire (Brăila), anonyme bourg provincial (les critiques croient y reconnaître Dorohoi, le lieu de naissance de l'écrivain). Dans tous les cas, la ville survit exclusivement à travers le ghetto, ce dernier changeant la vision de la compréhension urbaine. Par sa position dans la géographie de la ville, le ghetto sera toujours marginal dans la prose de l'entredeux-guerres, et sa délimitation sera d'autant plus précise, lorsque la superficie de la ville est réduite. La fluidité des quartiers de la communauté juive de Bucarest chez Peltz ne se prolonge que partiellement dans le canevas des rues de Brăila, chez Ury Benador, elle manque pourtant chez Ion Călugăru, où, dans une description détaillée des espaces de vie et de leurs habitants pauvres, une fracturation imaginaire à la moitié de la rue délimite brutalement les Juifs de leurs voisins : «Ce n'est pas loin jusqu'au facteur, bien qu'il semble aux confins de la Terre. Car la rue est divisée en deux : une moitié, c'est-à-dire de l'usine de soda à la maison de la laitière Basia, habitée par des Juifs, et l'autre moitié jusqu'au pré, par les faubouriens $»$.

C'est sous le regard neutre d'un protagoniste âgé de cinq ans, Buium, que les principes qui organisent un shtet ${ }^{39}$ traditionnel du nord de la Moldavie sont recomposés pour la décennie 1907-1917 : pauvreté désolante - faim - misère. Bien que la coexistence des Juifs avec d'autres ethnies, à l'intérieur du ghetto, oscille, chez Ion Călugăru, entre tolérance relative et ignorance totale, les allusions aux événements de 1907 (quand plusieurs attaques sont enregistrées contre des membres de la communauté) accentuent leur vulnérabilité, leur isolement, enfin, leur résignation devant le danger extérieur. Un autre moment antisémite, enregistré quelques décennies plus tard et évoqué par Peltz dans L'Avenue Văcărești, surprend la communauté armée et décidée de défendre à tout prix son quartier devant la menace venant, désormais, de l'intérieur de la ville ; la ségrégation spatiale se réalise, cette fois, par les zones envahies par des «hommes en vêtements verts $»^{40}$ et de leurs sympathisants, "marchands des quatre saisons et faubouriens » («d'Obor et Belu, de Philanthropie et des fosses de Ouatu, de Ștefan cel Mare et Jianu »).

Chez Ury Benador, la ville de Dorohoi est un personnage plutôt absent, avec un minimum d'éléments indiqués de sa géographie urbaine : le terrain vague de la foire, dans le voisinage de la maison louée par la famille du tailleur Mendl Landau,

\footnotetext{
${ }^{39}$ Terme désignant, en Europe de l'Est, une localité urbaine à population majoritaire juive.

${ }^{40}$ Les membres du parti fasciste roumain, «La Légion de l'Archange Michel» (1927-1941), dénommés « légionnaires » ou " chemises vertes » à cause de leur uniforme.
} 
et la gare d'où elle part pour Brăila. Aucune image commune, cependant, avec celles évoquées par Ion Călugăru. La richesse de la vie spirituelle de ce qui fut autrefois le shtetl, récupérable largement chez Peltz, dans L'Avenue Văcărești, est placée, chez Ion Călugăru, dans L'enfance d'un vaurien, en arrière-plan, parmi les rares repères en ce sens restant la préoccupation constante pour l'éducation de l'enfant Buiumaş.

Si Ury Benador reconstruit le ghetto de Brăila de la périphérie au centre, à l'aide de son personnage Mendl Landau qui forçe le centre, pour lui l'équivalent de la péripherie étant la pauvreté, la maladie, la disparition et, au pôle opposé, le succès, la prospérité, le respect, Peltz l'assemble de «l'intérieur», de la maison surpeuplée de la couturière Esther, insalubre et misérable, où l'auberge, dont elle fait partie, n'est qu'une réplique en miniature du ghetto.

Après tout, l'interrogation rhétorique de la grand-mère Leia : «Mais qui est heureux dans l'auberge? Qui y est vraiment heureux ?», ne présume pas une réponse affirmative de la part d'aucun de ses nombreux locataires ${ }^{41}$, ni même de la part des autres, à l'extérieur d'elle, "mendiants, affamés, vaincus par la vie »; à l'exception, peut-être, des personnages extraits de la petite bourgeoisie de la même communauté, qui bougent dans le roman Feu à l'Auberge aux Tilleuls. La suspension du spectaculaire, ancrée dans le quotidien fruste, mais aussi l'annulation de toute intrigue significative fait de L'Avenue Văcărești « une chronique de l'existence anodine de la communauté juive dans les faubourgs de Bucarest $»^{42}$.

À travers le drame multiplié (celui de l'équité, de la dévotion, le drame de l'impuissance, de la survie - et Peltz est, à cet égard, un comptable assidu) et le supplice sisyphéen de ne rien faire d'important ou de définitif, la petite communauté de l'auberge (et, à travers elle, L'Avenue Văcărești), personnifie une forme de résistance héroïque par le simple fait de survivre. Extrêmement rare, le cours prédestiné de la communauté est perturbé par une abdication temporaire du « devoir » (c'est le cas du personnage qui vend des craquelins ronds et qui refuse, un beau jour, devant ses garçons "pétrifiés par la peur », de vendre ses produits), ou par une abdication permanente (le cas de la famille du tailleur Mendl qui choisit le néant, intoxiquée au manganèse). Interprété uniquement comme une démission et une condamnation définitive, le geste final du descendant sensible et surdoué d'Esther de se marier et de rester dans le ghetto, alors que tout le monde le croyait capable de sortir de cet environnement, pourrait être considéré, à son tour, comme son refus d'abdication... au devoir. Les tribulations de l'auteur dans des différents quartiers, reconstituées par Rodica Lăzărescu, dans la monographie qu'elle

\footnotetext{
${ }^{41}$ Madame Sura avec son fils Alfred, le tailleur Feldman avec les six enfants, la veuve Katz, la famille de l'artiste Huna, avec huit enfants, le vendeur de craquelins ronds avec dix garçons, la femme pianiste avec son mari paralysé, la famille d'un ancien combattant de cirque.

${ }^{42}$ Ovidiu Morar, Scriitori evrei..., p. 125.
} 
consacre à Peltz, Viața cu haz și fără a numitului Peltz [La vie avec et sans humour du nommé Peltz], et son retour définitif dans la communauté, où il s'impliquera en aidant les plus humbles, converge vers une interprétation similaire.

L'attention au détail, la reconstruction du quotidien, la reprise du fait anodin, l'effort de récupérer la vie communautaire juive de l'entre-deux-guerres dans son intégralité, représentent la caractéristique la plus précieuse du roman du ghetto roumain. Néanmoins, on ne connait toujours pas beaucoup sur la Shoah roumaine. En raison de son objectivité, L'Avenue Văcărești de Peltz reste parmi les sources les plus crédibles dans la reconstruction des quartiers juifs des villes roumaines, aujourd'hui disparus.

\section{BIBLIOGRAPHIE}

BAHTIN, M., Probleme de literatură şi estetică [Problèmes d'esthétique et de littérature]. Traduit par Nicolae Iliescu. Préface de Marian Vasile, București, Univers, 1982.

BALTAZAR, Camil, « De vorbă cu d-l I. Peltz. Cu prilejul apariției romanului Calea Văcărești» [«Entretien avec M. I. Peltz, à l'occasion de la publication du roman L'Avenue Văcărești »], România literară, 2, 1933, 87, pp. 3-4.

BENADOR, Ury, Ghetto Veac XX [Ghetto XX Siècle], București, Editura Librăriei «Universala » Alcalay \& Co, [1934].

CĂLINESCU, G., Istoria literaturii române de la origini până în prezent [L'Histoire de la littérature roumaine dès les origines jusqu'au présent]. Édition et preface d'Al. Piru, București, Minerva, 1982.

CĂLUGĂRU, Ion, Lumina primăverii [La lumière du printemps], București, Socec, [1939].

CALUGARU, Ion, Trustul [Le Trust], [București], Editura « Națională Ciornei », [1937].

CONSTANTINESCU, Pompiliu, Scrieri [Écrits], București, Editura pentru Literatură, 1967.

CRĂCIUN, Camelia, «Représentations de la vie et de l'espace juifs dans la littérature roumaine de l'entre-deux-guerres. Le Bucarest juif reflété dans les œuvres d'Isac Peltz », Etudes Balkaniques, 2010, 17, pp. 85-105.

LĂZĂRESCU, Rodica, Viața cu haz și fără a numitului Peltz [La vie avec et sans humour du nommé Peltz], București, Academia Română, Fundația Națională pentru Știință și Artă, Institutul de Istorie și Teorie Literară « G. Călinescu », 2005.

Ludo, I., Ghettouri [Ghettos], București, « Cartea », 1939.

MALINOVICH, Nadia, «Littérature populaire et romans juifs dans la France des années 1920 », Archives Juifs, 39, 2006, 1, pp. 46-62.

MORAR, Ovidiu, Scriitori evrei din România [Ecrivains juifs de Roumanie], București, Hasefer, 2014.

OIȘTEANU, Andrei, Imaginea evreului în cultura română. Studiu de imagologie în context estcentral-european [L'image du Juif dans la culture roumaine. Étude d'imagologie dans un contexte d'Europe centrale et orientale], III İme édition, révisée et illustrée, Iași, Polirom, 2012.

PELTZ, I., Calea Văcărești [L'Avenue Văcărești], București, ESPLA, 1957.

PODEANU, I., « Douăzeci de minute cu d. I. Peltz » [« Vingt minutes avec M. I. Peltz »], Rampa, 17, 1934, 5001, p. 1.

ȘĂINEANU, Lazăr, Dicționar universal al limbei române [Dictionnaire universel de la langue roumaine], IV ème édition, Craiova, Scrisul Românesc, 1929. 
SCRIBAN, August, Dicționarul limbii românești [Le dictionnaire de la langue roumaine], București, Institutul de Arte Grafice « Presa Bună », 1939.

VERGATTI, Radu Ștefan, « Dinamica demografică și aspecte socio-profesionale ale obștii evreiești din București (1810-1939)» [« La dynamique démographique et aspects socioprofessionnels de la communauté juive de Bucarest (1810-1939)»], Revista de Istorie Socială, 1997-1998, 2-3, pp. $165-235$.

WACQUANT, Loïc, "Repenser le ghetto. Du sense commun au concept sociologique », Idées économiques et sociales, 2012, 167, pp. 14-25.

WIRTH, Louis, Ghetoul [Le Ghetto]. Traduit par Lăcrămioara Juverdeanu. Étude introductive de Mihai Dinu Gheorghiu, Iași, Editura Universității « Al. I. Cuza », 2012.

ZISSU, A.L., « Literatura evreiască din România » [« La littérature juive de Roumanie »], Adam, 3, 1931, 38, pp. 10-12.

\section{THE GHETTO IN THE ROMANIAN INTER-WAR NOVEL} (Abstract)

Two years pass between 1931, when A.L. Zissu, one of the most important representatives of the Jewish community in Romania, voices publicly his doubt regarding the existence of the Romanianlanguage Jewish literature, and the moment of publication of the volume opening the series of the ghetto-illustrating novels (I. Peltz, Calea Văcărești, 1933). By the time of the political rise of the radicalized right wing, which concluded with the ostracizing of the Jewish community, and, in the cultural sector, with the blacklisting of their literary creation, Foc in Hanul cu Tei [Fire in the Linden Tree Inn] (1934) by the same I. Peltz, Ghetto Veac XX [20 ${ }^{\text {th }}$ Century Ghetto] by Ury Benador, and Copilăria unui netrebnic [A Wretched Man's Childhood] (1936) by Ion Călugăru were published. From the Văcărești Route of Greater Romania's growing metropolis, through the Jewish neighborhood of an important town by the Danube (Brăila), to "ulicioara Țiprei" in the anonymous Moldavian bourg of Dorohoi, the ghetto novel tends to complete out of time the fresco of the interwar Jewish community; the scope of this phenomenon can be guessed by looking at the started literary projects, some of them abandoned after the first volume (Ury Benador's trilogy, Ghetto veac $X X)$, some other completed (continuing Copilăria unui netrebnic, Ion Călugăru writes Trustul [The Trust], 1937, and Lumina primăverii [The Light of Spring], 1939). From the very beginning, the frequency of the phrase in the literary text is insignificant, despite the fact that it has been present in the Romanian prose writing since the third decade of the $20^{\text {th }}$ century in two of the titles, at Benador (Ghetto veac $X X)$ and Ludo (Ghettouri $[$ Ghettos]). Here, too, the ghetto refers to its historic meaning of elsewhere, its local pinpointing being challenging; and when referenced, it is done metaphorically, linked with a state of sentimental isolation, at I. Peltz and Ury Benador. By the time of the terrible connotations it would acquire in the following decade, the "ghetto" painted by the Romanianlanguage Jewish writers would grow perceptively close to what Loïc Wacquant calls the "ethnic neighborhood", and the idea is backed by a flexibility of its boundaries and an incipient intrinsic cultural diversity. Commitments such as the rejection of the artificial, objectivity, personal experience guide the Jewish writer's narrative endeavor in the temporal cut-out of the third decade: overall, the ghetto novel resounds, first of all, owing to its biographic nature, while some other particularities include the "harmony of space", by keeping the plot within the exclusive perimeter of the ghetto and by anchoring it in everyday life.

Keywords: marginal space, minority community, ghetto, shtetl, everyday reality. 


\section{GHETOUL ÎN ROMANUL ROMÂNESC INTERBELIC (Rezumat)}

Între momentul îndoielii exprimate public privind existența unei literaturi evreiești de limbă română de către unul dintre cei mai importanți reprezentanți ai comunității evreiești din România, A.L. Zissu, în 1931, și apariția volumului ce deschide suita romanelor reprezentative ale ghetoului (I. Peltz, Calea Văcărești, 1933) se succed doi ani. Până la ascensiunea politică a dreptei radicalizate, culminând cu ostracizarea populații evreiești, iar în plan cultural, cu punerea la index a creației ei literare, vor apărea Foc în Hanul cu Tei (1934) al aceluiași I. Peltz, Ghetto Veac XX, al lui Ury Benador și Copilăria unui netrebnic (1936) de Ion Călugăru. Din Calea Văcărești a metropolei în plină emancipare a României Mari, prin cartierul evreiesc al unui important oraș dunărean (Brăila), la „ulicioara Țiprei” din anonimul târg moldav al Dorohoiului, romanul ghetoului tinde să întregească fresca comunității evreiești interbelice în contratimp, fenomen a cărui anvergură e intuibilă din proiectele literare demarate, câteva abandonate după primul volum (trilogia lui Ury Benador, Ghetto veac $X X$ ), altele, definitivate (în continuarea Copilăriei unui netrebnic, Ion Călugăru semnează Trustul, 1937 și Lumina primăverii, 1939). Chiar din debut, sintagma face dovada unei frecvențe nesemnificative în textul literar, deși prezentă în proza românească din deceniul trei al veacului XX în două dintre titluri, la Benador (Ghetto veac XX) și Ludo (Ghettouri). Şi aici, ghetoul trimite, mai curând, la semnificația sa istorică, de aiurea, dificil reperabilă local, și atunci metaforic, asociat claustrării sentimentale, la I. Peltz și Ury Benador. Până la valențele atroce ale deceniului următor, „ghetoul" scriitorilor evrei de limbă română se apropie sensibil de ceea ce Loïc Wacquant numea „cartier etnic”, în susținerea ideii venind o flexibilitate a frontierelor sale şi o incipientă diversitate culturală în interior. Angajamente precum respingerea artificialului, obiectivitatea, experiența personală ghidează demersul narativ al scriitorului evreu în decupajul temporal al deceniului trei: privit, în ansamblu, romanul ghetoului convinge, în primul rând, prin caracterul său biografic, printre alte particularități aflându-se „unitatea spațiului”, prin menținerea tramei în perimetrul exclusiv al ghetoului și ancorarea în cotidian.

Cuvinte-cheie: spațiu marginal, comunitate minoritară, ghetou, ștetl, realitate cotidiană. 